\title{
Moment Closure Techniques for Stochastic Models in Population Biology
}

\author{
Abhyudai Singh and João Pedro Hespanha
}

\begin{abstract}
Continuous-time birth-death Markov processes serve as useful models in population biology. In this paper, we present a procedure for constructing approximate stochastic models for these processes. This is done by representing the population of a specie as the continuous state of a Stochastic Hybrid System (SHS). This SHS is characterized by reset maps that account for births and deaths, transition intensities that correspond to the birth-death rates, and trivial continuous dynamics. It has been shown that for this type of SHS the statistical moments of the continuous state evolve according to an infinite-dimensional linear ordinary differential equation (ODE). However, for analysis purposes it is convenient to approximate this infinite-dimensional linear ODE by a finite-dimensional nonlinear one. This procedure generally approximates some higher-order moments by a nonlinear function of lower-order moments and it is called moment closure.
\end{abstract}

We obtain moment closures by matching time derivatives of the infinite-dimensional ODE with its finite-dimensional approximation at some time $t_{0}$. This guarantees a good approximation, at-least locally in time. We provide explicit formulas to construct these approximations and compare this technique with alternative moment-closure methods available in the literature. This comparison takes into account both the transient and the steady-state regimens.

\section{INTRODUCTION}

Continuous-time birth-death Markov processes have been extensively used for modeling stochasticity in population biology [1], [2], [3]. The time evolution of this process is typically described by a single equation for a grand probability function, where time and species populations appear as independent variables, called the Master or Kolmogorov equation [4]. However, this equation can only be solved for relatively few, highly idealized cases and a more reasonable goal is to determine the evolution of a few low-order moments.

In this paper, a method for estimating lower-order moments is introduced for a Markov process involving a single specie, with birth and death rates being polynomials of order $s \in \mathbb{N}_{\geq 2}$. This process is modeled by a Stochastic Hybrid System (SHS) whose state $\mathbf{x}$ is the population of the specie. This SHS has trivial continuous dynamics $\dot{\mathbf{x}}=0$,

This material is based upon work supported by the Institute for Collaborative Biotechnologies through grant DAAD19-03-D-0004 from the U.S. Army Research Office and by the National Science Foundation under Grant No. CCR-0311084.

A.Singh and J.P.Hespanha are with the Center for Control Engineering and Computation University of California, Santa Barbara, CA 93101. abhidengineering.ucsb.edu, hespanha@ece.ucsb.edu
TABLE I

SEPARABLE DERIVATIVE-MATCHING MOMENT CLOSURE FUNCTION $\varphi_{\ell}^{s}(v), \ell \in\{n+1, \ldots, n+4\}$.

\begin{tabular}{ccccc}
\hline & $\ell=n+1$ & $\ell=n+2$ & $\ell=n+3$ & $\ell=n+4$ \\
\hline$n=2$ & $\frac{v_{2}^{3}}{v_{1}^{3}}$ & $\frac{v_{2}^{6}}{v_{1}^{8}}$ & $\frac{v_{2}^{10}}{v_{1}^{15}}$ & $\frac{v_{2}^{15}}{v_{1}^{24}}$ \\
$n=3$ & $\frac{v_{1}^{4} v_{3}^{4}}{v_{2}^{6}}$ & $\frac{v_{1}^{15} v_{3}^{10}}{v_{2}^{20}}$ & $\frac{v_{1}^{36} v_{3}^{20}}{v_{2}^{45}}$ & $\frac{v_{1}^{70} v_{3}^{35}}{v_{2}^{84}}$ \\
$n=4$ & $\frac{v_{2}^{10} v_{4}^{5}}{v_{1}^{5} v_{3}^{10}}$ & $\frac{v_{2}^{45} v_{4}^{15}}{v_{1}^{24} v_{3}^{40}}$ & $\frac{v_{2}^{126} v_{4}^{35}}{v_{1}^{70} v_{3}^{105}}$ & $\frac{v_{2}^{280} v_{4}^{70}}{v_{1}^{160} v_{3}^{224}}$
\end{tabular}

and is characterized by reset maps that account for births and deaths and transition intensities that correspond to the birth-death rates. Details of the Markov process and its stochastic modeling are presented in Section II. Similar SHS were used in [5] for the stochastic modeling of single specie elementary chemical reactions, however, in this case the transitional intensities are always polynomials of order 2.

Let $\mu_{m}$ be the $m^{\text {th }}$ order moment of $\mathbf{x}$. We will show in Section III that the time derivative of $\mu_{m}$ is a linear combination of moments upto order $m+s-1$. Thus, if one creates an infinite vector containing all the statistical moments of $\mathbf{x}$, the dynamics of this vector is governed by an infinite-dimensional linear ordinary differential equation (ODE) which we call the infinite-dimensional moment dynamics. However, for analysis purposes it is convenient to approximate this infinite-dimensional linear ODE by a finite-dimensional nonlinear one, which we refer to as the truncated moment dynamics and denote its state by $v=\left[v_{1}, \ldots, v_{n}\right]^{T}$. As the dynamics of vector $\mu=\left[\mu_{1}, \ldots, \mu_{n}\right]^{T}$ is given by

$$
\dot{\mu}=A \mu+B\left[\mu_{n+1}, \ldots, \mu_{n+s-1}\right]^{T},
$$

for some matrices $A$ and $B$, this procedure generally approximates the moments $\mu_{\ell}, \ell \in\{n+1, \ldots, n+s-1\}$ by a nonlinear function $\varphi_{\ell}(v)$ of $v_{1}, \ldots, v_{n}$, which we call the moment closure function. With this the truncated moment dynamics can be written as

$$
\dot{v}=A v+B\left[\varphi_{n+1}(v), \ldots, \varphi_{n+s-1}(v)\right]^{T} .
$$

In Section IV, we consider moment closure functions of the 
following separable form:

$$
\varphi_{\ell}^{s}(v)=\prod_{m=1}^{n} v_{m}^{\gamma_{m}^{\ell}}
$$

for appropriately chosen constants $\gamma_{m}^{\ell} \in \mathbb{R}$. The constants are obtained by matching time derivatives of the infinitedimensional ODE with its finite-dimensional approximation at some time $t_{0}$. We refer to this moment closure as the separable derivative-matching moment closure. For truncation of order $n=\{2,3,4\}$ and $s=5$ this procedure leads to the formulas in Table I.

Moment closure methods which have been used in literature, typically achieve moment closure by assuming the probability distribution to be normal [6], lognormal [7], poisson and binomial [8]. We refer to them as normal, lognormal, poisson and binomial moment closures respectively. In Section V, they are compared with the separable derivative-matching moment closure, for a special class of the birth-death Markov process known as the stochastic logistic model.

In Section V-A the comparisons are done based on how well the time derivatives of the moment closure function $\varphi_{\ell}(v)$ match those of the higher-order moment $\mu_{\ell}$. We show in Section V-A, that with the exception of the poisson, all the moment closure achieve approximate derivative matching. We propose an alternative poisson moment closure function, which unlike its previous counterpart, does match derivatives approximately, and is subsequently shown to perform better.

In Section V-B comparison are done based on the steady-state solutions of the truncated moment dynamics. We show that the separable derivative-matching moment closure, always yields a unique non-trival positive real steady-state $\forall n \in \mathbb{N}_{\geq 2}$. Thus, it is in some sense superior to the other moment closures, which can have spurious, imaginary and even stable negative steady-states, and hence, biologically meaningless.

\section{Jump Markov Process Model}

We consider a continuous time birth-death Markov process, where the conditional probabilities of a unit increase and decrease, respectively, in an "infinitesimal" time interval $(t, t+d t]$ is given by

$$
\begin{aligned}
& \mathrm{P}\{\mathbf{x}(t+d t)=x+1 \mid \mathbf{x}(t)=x\}=\left\{\begin{array}{c}
\eta(x) d t, \forall x \leq U \\
0,
\end{array}\right. \\
& \mathrm{P}\{\mathbf{x}(t+d t)=x-1 \mid \mathbf{x}(t)=x\}=\chi(x) d t,
\end{aligned}
$$

where

$$
\eta(x):=a_{0}+a_{1} x+\ldots+a_{s} x^{s}, \quad \chi(x):=b_{1} x+\ldots+b_{s} x^{s}
$$

for some integer $s \geq 2, U \in \mathbb{N}_{\geq 0}$ and

$$
a_{0} \geq 0, \quad \chi(x)>0, \quad \eta(x)>0, \quad \forall x \in(0, U), \quad \eta(U)=0 .
$$

We assume that the initial condition satisfies $\mathbf{x}\left(t_{0}\right) \in[0, U]$, and hence, $\mathbf{x}(t) \leq U, \forall t \in[0, \infty)$ with probability one. We call $U$ as the population limit.

To model the time evolution of $\mathbf{x}(t)$, we consider a special class of Stochastic Hybrid Systems (SHS), which was introduced in [9] to model the stochastic time evolution of the populations of different species involved in a chemical reaction. More specifically, to fit the framework of our problem, these system are characterized by trivial dynamics

$$
\dot{\mathbf{x}}=0,
$$

two reset maps

$$
\mathbf{x} \mapsto \phi_{1}(\mathbf{x}):=\mathbf{x}+1, \quad \mathbf{x} \mapsto \phi_{2}(\mathbf{x}):=\mathbf{x}-1
$$

and the corresponding transition intensities

$$
\lambda_{1}(\mathbf{x}):=\eta(x), \quad \lambda_{2}(\mathbf{x}):=\chi(\mathbf{x}) .
$$

In essence, if no "event" takes place, the state remains constant and whenever a "birth event" or "death event" takes place, the corresponding $\phi_{i}(\mathbf{x})$ is "activated" and $\mathbf{x}$ is reset accordingly, furthermore, the probability of the activation taking place in an "infinitesimal" time interval $(t, t+d t]$ is $\lambda_{i}(\mathbf{x}) d t$.

\section{Moment Dynamics}

Ideally, one would like to determine the evolution of the probability distribution for $\mathbf{x}(t)$. In general, this is difficult and a more reasonable goal is to determine the evolution of a few low-order moments.

Given $m \in \mathbb{N}_{\geq 1}$, we define the $m^{\text {th }}$ order (uncentered) moment of $\mathbf{x}$ to be

$$
\mu_{m}(t)=\mathbf{E}\left[\mathbf{x}(t)^{m}\right], \quad \forall t \geq 0,
$$

where $\mathbf{E}$ stands for the expected value. The time evolution of moments is given by the following result, which is a straight forward application of Theorem 1 in [10] to the SHS (3)-(5).

Theorem 1: For every continuously differentiable function $\psi: \mathbb{R} \rightarrow \mathbb{R}$ we have that

$$
\begin{aligned}
\frac{d \mathbf{E}[\psi(\mathbf{x})]}{d t} & =\mathbf{E}[(\mathbf{L} \psi)(\mathbf{x})], \\
(\mathbf{L} \psi)(\mathbf{x}) & =\sum_{i=1}^{2}\left[\psi\left(\phi_{i}(\mathbf{x})\right)-\psi(\mathbf{x})\right] \lambda_{i}(\mathbf{x}) .
\end{aligned}
$$

The operator $\psi \mapsto \mathbf{L} \psi$ defined by (8) is called the extended generator of the SHS.

Since the reset maps $\phi_{i}(\mathbf{x})$ and transitional intensities $\lambda_{i}(\mathbf{x})$ are polynomials in $\mathbf{x}$, the extended generator $(\mathbf{L} \psi)(\mathbf{x})$ is a polynomial in $\mathbf{x}$ for every polynomial $\psi(\mathbf{x})$ in $\mathbf{x}$. Such SHSs whose extended generator $\mathbf{L}$ is closed on the set of polynomials in $\mathbf{x}$ are called polynomial Stochastic Hybrid System (pSHS). 
With $\psi(\mathbf{x})=\mathbf{x}^{m}$ in (7), we have from (8) that

$$
(\mathbf{L} \psi)(\mathbf{x})=\eta(\mathbf{x})\left[(\mathbf{x}+1)^{m}-\mathbf{x}^{m}\right]+\chi(\mathbf{x})\left[(\mathbf{x}-1)^{m}-\mathbf{x}^{m}\right] .
$$

Using (2) and Theorem 1, we have that the evolution of $\mu_{m}$, $\forall m \in \mathbb{N}_{\geq 1}$ can be written as

$$
\dot{\mu}_{m}=\sum_{p=0}^{s} \sum_{r=0}^{m+s-1} \mathrm{C}_{m+p-r}^{m} f(m+p-r, p) \mu_{r}
$$

where $\mu_{0}=1, \mathrm{C}_{j}^{m}$ is defined as follows ${ }^{1} \forall j, m \in \mathbb{N}$

$$
\begin{aligned}
\mathrm{C}_{j}^{m}: & = \begin{cases}\frac{m !}{(m-j) ! j !} & m \geq j \geq 0 \\
0 & m<j\end{cases} \\
f(j, p) & := \begin{cases}0 & j=0 \\
a_{1}+(-1)^{j} a_{2} & j>0, p=1 \\
-b_{1}+(-1)^{j} b_{2} & j>0, p=2 .\end{cases}
\end{aligned}
$$

One can see from the right-hand-side of (9), that the time derivative of $\mu_{m}$ is a linear combination of moments $\mu_{r}$, upto order $r=m+s-1$. Hence, if one stacks all moments in an infinite vector $\mu_{\infty}=\left[\mu_{1}, \mu_{2}, \cdots\right]^{T}$, its dynamics can be written as

$$
\dot{\mu}_{\infty}=A_{\infty} \mu_{\infty}
$$

for some infinite matrix $A_{\infty}$. We refer to (12) as the infinitedimensional moment dynamics. Let $\mu=\left[\mu_{1}, \mu_{2}, \ldots, \mu_{n}\right]^{T} \in$ $\mathbb{R}^{n}$ contains the top $n$ elements of $\mu_{\infty}$. Then, using (9) the evolution of $\mu$ is given by

$$
\dot{\mu}=A \mu+B \bar{\mu}, \quad \bar{\mu}=\left[\mu_{n+1}, \ldots, \mu_{n+s-1}\right]^{T},
$$

for some $n \times n$ and $n \times s-1$ matrices $A$ and $B$. Our goal is to approximate (13) by a finite-dimensional nonlinear ODE of the form

$$
\begin{aligned}
& \dot{v}=A v+B \bar{\varphi}(v), \quad v=\left[v_{1}, v_{2}, \ldots, v_{n}\right]^{T} \\
& \bar{\varphi}(v)=\left[\varphi_{n+1}(v), \ldots, \varphi_{n+s-1}(v)\right]^{T}
\end{aligned}
$$

where the map $\bar{\varphi}: \mathbb{R}^{n} \rightarrow \mathbb{R}^{s-1}$ should be chosen so as to keep $v(t)$ close to $\mu(t)$. We call (14) the truncated moment dynamics and $\varphi_{\ell}(v)$ the moment closure function for $\mu_{\ell}$, $\forall \ell \in\{n+1, \ldots, n+s-1\}$.

When a sufficiently large but finite number of derivatives of $\mu(t)$ and $v(t)$ match point-wise, then, the difference between solutions to (13) and (14) remains close on a given compact time interval. This follows from a Taylor series approximation argument. To be more precise, for each $\delta>0$ and integer $N$, there exists $T \in \mathbb{R}$, for which the following result holds: Assume that for every $t_{0} \geq 0$,

$\mu\left(t_{0}\right)=\left.v\left(t_{0}\right) \Rightarrow \frac{d^{i} \mu(t)}{d t^{i}}\right|_{t=t_{0}}=\left.\frac{d^{i} v(t)}{d t^{i}}\right|_{t=t_{0}}, \forall i \in\{1, \ldots, \mathrm{N}\}$

\footnotetext{
${ }^{1} n$ ! denotes the factorial of $n$.
}

where $\frac{d^{i} \mu(t)}{d t^{i}}$ and $\frac{d^{i} v(t)}{d t^{i}}$ represent the $i^{t h}$ time derivative of $\mu(t)$ and $v(t)$ along the trajectories of system (12) and (14) respectively. Then,

$$
\mu\left(t_{0}\right)=v\left(t_{0}\right) \Rightarrow\|\mu(t)-v(t)\| \leq \delta, \forall t \in\left[t_{0}, T\right],
$$

along solutions of (12) and (14), where $\mu$ denote the first $n$ elements of $\mu_{\infty}$. It has been shown in [11] that under appropriate asymptotic stability conditions on (12), the inequality (16) can actually be extended $\forall t \in\left[t_{0}, \infty\right)$. In the next section we will use (15) to construct moment closure functions $\varphi_{\ell}(v)$.

\section{Separable Derivative-Matching Moment Closures}

In this section we construct truncated moment dynamics (14) for the class of birth-death Markov processes introduced in Section II using (15). After replacing (13) and (14) in (15), equality (15) becomes a PDE on $\bar{\varphi}$. We will seek for solutions $\bar{\varphi}$ to this PDE, whose entries have the following separable form

$$
\begin{aligned}
& \varphi_{\ell}^{s}(v)=\prod_{m=1}^{n} v_{m}^{\gamma_{m}^{\ell}}:=v^{\left(\gamma_{\ell}\right)}, \quad \gamma_{\ell}=\left(\gamma_{1}^{\ell}, \ldots, \gamma_{n}^{\ell}\right) \\
& \forall v=\left[v_{1}, \ldots, v_{n}\right]^{T}, \quad \forall \ell \in\{n+1, \ldots, n+s-1\}
\end{aligned}
$$

for appropriately chosen constants $\gamma_{m}^{\ell} \in \mathbb{R}$. In the sequel we will refer to such $\varphi_{\ell}^{s}(v)$ as a Separable Derivative-Matching (SDM) moment closure function for $\mu_{\ell}$. Often, it is not possible to find $\bar{\varphi}(v)$ of the form (17) for which (15) holds exactly. We will therefore relax this condition and simply demand the following

$$
\mu\left(t_{0}\right)=\left.v\left(t_{0}\right) \Rightarrow \frac{d^{i} \mu(t)}{d t^{i}}\right|_{t=t_{0}}=\left.\frac{d^{i} v(t)}{d t^{i}}\right|_{t=t_{0}}+\mathbf{E}\left[\varepsilon_{i}\left(\mathbf{x}\left(t_{0}\right)\right)\right]
$$

$\forall i \in\{1,2\}$, where each element of the vector $\varepsilon_{i}\left(\mathbf{x}\left(t_{0}\right)\right)$ is a polynomial in $\mathbf{x}\left(t_{0}\right)$. One can think of (18) as an approximation to (15) which will be valid as long as $\left.\frac{d^{i} \mu(t)}{d t^{i}}\right|_{t=t_{0}}$ dominate $\mathbf{E}\left[\varepsilon_{i}\left(\mathbf{x}\left(t_{0}\right)\right)\right]$.

The following theorem, the proof of which is omitted due to space considerations, summarizes the main result.

Theorem 2: Let $\gamma_{\ell}=\left(\gamma_{1}^{\ell}, \ldots, \gamma_{n}^{\ell}\right), \forall \ell \in\{n+1, \ldots, n+s-1\}$ be chosen as the unique solution of the following system of linear equations

$$
\mathrm{C}_{k}^{\ell}=\sum_{m=1}^{n} \gamma_{m}^{\ell} \mathrm{C}_{k}^{m}, \quad \forall k=\{1, \ldots, n\}
$$

Then, for every deterministic initial condition $\mu_{\infty}\left(t_{0}\right)=$ $\left[x_{0}, x_{0}^{2}, \ldots\right]^{T}$ which corresponds to $\mathbf{x}\left(t_{0}\right)=x_{0}$ with probability one, we have

$$
\mu\left(t_{0}\right)=v\left(t_{0}\right) \Rightarrow\left\{\begin{array}{c}
\left.\frac{d \mu(t)}{d t}\right|_{t=t_{0}}=\left.\frac{d v(t)}{d t}\right|_{t=t_{0}} \\
\left.\frac{d^{2} \mu(t)}{d t^{2}}\right|_{t=t_{0}}=\left.\frac{d^{2} v(t)}{d t^{2}}\right|_{t=t_{0}}+\varepsilon_{2}\left(x_{0}\right),
\end{array}\right.
$$


where $\frac{d^{i} \mu(t)}{d t^{i}}$ and $\frac{d^{i} v(t)}{d t^{i}}$ represent the $i^{t h}$ time derivative of $\mu(t)$ and $v(t)$ along the trajectories of system (12) and (14) respectively and the $m^{\text {th }}$ element of the vector $\varepsilon_{2}\left(x_{0}\right)$ is a polynomial in $x_{0}$ of order $m-n+2(s-1)$, for $m-n+2(s-1) \geq s$ and equal to zero otherwise.

Remark 1. Using (9) it can be shown that $\frac{d^{2} \mu_{m}(t)}{d t^{2}}$ is a linear combination of moments of $\mathbf{x}$ upto order $m+2(s-1)$. Thus, $\left.\frac{d^{2} \mu_{m}(t)}{d t^{2}}\right|_{t=t_{0}}$ is a polynomial in $x_{0}$ of order $m+2(s-1)$, and hence, for ${ }^{2} x_{0}>>1, \varepsilon_{2}^{m}\left(x_{0}\right) /\left.\frac{d^{2} \mu_{m}(t)}{d t^{2}}\right|_{t=t_{0}}=\mathscr{O}\left(x_{0}^{-n}\right)$, where $\varepsilon_{2}^{m}\left(x_{0}\right)$ is the $m^{t h}$ element of $\varepsilon_{2}\left(x_{0}\right)$. Hence, with increasing $n$ or $x_{0}$, the truncated moment dynamics model should provide a more accurate approximations to the lower order moments.

Remark 2. One can see from (19) that the constants $\gamma_{m}^{\ell}$ are independent of the coefficient of the polynomials in (2). Moment closures for different values of $\ell$ that satisfy (19) are listed in Table I. Moreover, one can check that the moment closure for $\mu_{\ell}$ as given by $\varphi_{\ell}^{s}(v)$ in Table $\mathrm{I}$ is consistent with $\mathbf{x}(t)$ being lognormally distributed.

Remark 3. Using Mathematica to perform the symbolic manipulations, we verified that the separable derivativematching moment closures also matches derivatives of order higher than 2 in (20). To be more precise, for $n \in\{2,3,4\}$ and $i \in\{2, \ldots, 9\}$, we have

$$
\mu\left(t_{0}\right)=v\left(t_{0}\right) \Rightarrow\left\{\begin{array}{l}
\left.\frac{d \mu(t)}{d t}\right|_{t=t_{0}}=\left.\frac{d v(t)}{d t}\right|_{t=t_{0}} \\
\left.\frac{d^{i} \mu(t)}{d t^{i}}\right|_{t=t_{0}}=\left.\frac{d^{i} v(t)}{d t^{i}}\right|_{t=t_{0}}+\varepsilon_{i}\left(x_{0}\right),
\end{array}\right.
$$

where the $m^{\text {th }}$ element of $\varepsilon_{i}\left(x_{0}\right)$ is a polynomial in $x_{0}$ of order $m-n+i(s-1)$, for $m-n+i(s-1) \geq s$ and equal to zero otherwise. We conjecture that the above equality holds $\forall n \in \mathbb{N}_{\geq 2}$ and $\forall i \in \mathbb{N}_{\geq 2}$ but we only verify for $n$ upto 4 and $i$ upto 9 .

\section{COMPARISON OF MOMEnT Closures}

In this section we compare the SDM moment closure with other moment closure techniques that have appeared in literature. We restrict our attention to a class of birth-death Markov processes, known as the stochastic logistic model. The stochastic logistic model is the stochastic birth-death analogous model of the well-known deterministic VerhulstPearl equations [12] and has been extensively used for modeling stochasticity in population biology [1], [2], [3]. In this model, the polynomials $\eta(\mathbf{x})$ and $\chi(\mathbf{x})$ in (2) are given by

$$
\eta(x):=a_{1} x-b_{1} x^{2}, \chi(x):=a_{2} x+b_{2} x^{2}, a_{1}>b_{1}, a_{1}>a_{2},
$$

where $a_{1}, a_{2}, b_{1}, \quad b_{2} \in \mathbb{R}_{>0}$ and the population limit $U=a_{1} / b_{1}$. For the stochastic logistic model we have $s=2$,

\footnotetext{
${ }^{2} \mathscr{O}($.$) denotes order of.$
}

TABLE II

DifFERENT MOMENT Closures FUnCTIONS FOR $\mu_{3}$

\begin{tabular}{lc}
\hline Technique & Moment Closure Function \\
\hline SDM & $\left(\frac{v_{2}}{v_{1}}\right)^{3}$ \\
Normal & $3 v_{2} v_{1}-2 v_{1}^{3}$ \\
Lognormal & $\left(\frac{v_{2}}{v_{1}}\right)^{3}$ \\
Nasell-Poisson & $v_{1}+3 v_{1} v_{2}-2 v_{1}^{3}$ \\
New -Poisson & $v_{2}-v_{1}^{2}+3 v_{1} v_{2}-2 v_{1}^{3}$ \\
Binomial & $2 \frac{\left(v_{2}-v_{1}^{2}\right)^{2}}{v_{1}}-\left(v_{2}-v_{1}^{2}\right)+3 v_{1} v_{2}-2 v_{1}^{3}$ \\
\hline
\end{tabular}

and hence, $\bar{\mu}=\mu_{n+1}$ in (13) and $\bar{\varphi}(v)=\varphi_{n+1}(v)$ in (14).

Most moment closure techniques that appeared in the literature start by assuming a specific distributions for the population, and use this assumption to express higher order moments as a function of the lower order ones. This has been done for well known classes of distributions, such as normal [6], lognormal [7], poisson and binomial [8] and for these distribution we simply say that $\varphi_{n+1}$ is the normal, lognormal, poisson, or binomial moment closure function. For a more detiled discussion on distribution based moment closures the reader is referred to [13].

For a second order truncation $(n=2)$, Table II lists these moment closure functions for $\mu_{3}$ along with the separable derivative matching (SDM) moment closure function. Nasell-Poisson refers to the poisson moment closure function proposed in [8], while New-poisson refers an alternative poisson moment closure function that we propose, which, as we will see, performs better than the one proposed in [8]. The explanation for this lies in the fact that the New-poisson has better derivative matching properties than the Nasell-Poisson, in the sense of (18).

In the sequel we use superscripts $s, l, g, p 1, p 2$ and $b$ to denote separable derivative-matching, lognormal, normal, Nasell-poisson, New-poisson and binomial moment closure functions, respectively. We introduce two criteria to compare the different moment closure techniques. The first is the error

$$
e(t)=\mu_{3}(t)-\varphi_{3}(v(t))
$$

and the second is the steady-state solution of the truncated moment dynamics. In particular we are especially interested in determining if there exists a unique non-trivial positive real steady-state which would be physically meaningfull. This is important, because the normal moment closure can have stable spurious, imaginary and sometimes even stable negative steady-states, which lead to biologically meaningless 
behaviour [7]. For space considerations, we will restrict our comparisons to a second order of truncation, i.e., $n=2$ only and refer the reader to [13] for a more general analysis. Also as from Table II we have $\varphi_{3}^{l}(v)=\varphi_{3}^{s}(v)$ for $n=2$ we do not need to discuss lognormal moment closure separately.

\section{A. Derivative matching}

For separable derivative-matching moment closure, the error (23) can be written as

$$
\begin{aligned}
e^{s}(t) & =\mu_{3}(t)-\varphi_{3}^{s}(v(t)) \\
& =\sum_{i=0}^{\infty} \frac{\left(t-t_{0}\right)^{i}}{i !}\left(\left.\frac{d^{i} \mu_{3}(t)}{d t^{i}}\right|_{t=t_{0}}-\left.\frac{d^{i} \varphi_{3}^{s}(v(t))}{d t^{i}}\right|_{t=t_{0}}\right) \\
& =\sum_{i=0}^{\infty} \frac{\left(t-t_{0}\right)^{i}}{i !} \varepsilon^{s}\left(i, 3, x_{0}\right)
\end{aligned}
$$

where

$$
\varepsilon^{s}\left(i, 3, x_{0}\right):=\left.\frac{d^{i} \mu_{3}(t)}{d t^{i}}\right|_{t=t_{0}}-\left.\frac{d^{i} \varphi_{3}^{s}(v(t))}{d t^{i}}\right|_{t=t_{0}}
$$

is called the derivative matching error. Similarly, by substituting $\varphi_{\ell}^{g}(v), \varphi_{\ell}^{p 1}(v), \varphi_{\ell}^{p 2}(v), \varphi_{\ell}^{b}(v)$ in (24)-(26), one can define the derivative matching error for other closures, which will be denoted using the appropriate superscripts.

With deterministic initial conditions $\mu_{\infty}\left(t_{0}\right)=\left[x_{0}, x_{0}^{2}, \ldots\right]^{T}$ as in Theorem 2, using Table II we have for $i=0$,

$\varepsilon^{s}\left(0,3, x_{0}\right)=\varepsilon^{g}\left(0,3, x_{0}\right)=\varepsilon^{p 2}\left(0,3, x_{0}\right)=\varepsilon^{b}\left(0,3, x_{0}\right)=0$,

$\varepsilon^{p 1}\left(0,3, x_{0}\right)=-x_{0}$.

For $i \geq 1$, using symbolic manipulation in Mathematica, we see that for each of these moment closures, the $i^{\text {th }}$ derivative matching error, is a polynomial in $x_{0}$ of order $i+1$, but with different coefficients. Hence, all moment closures match derivatives approximately, with the error being of the same order in $x_{0}$. Since $\varepsilon^{p 1}\left(0,3, x_{0}\right)=-x_{0}$, the Nasell-poisson moment closure will have a large initial error as compared with New-poisson moment closure. Simulation results show that with the exception of Nasell-poisson moment closure, which consistently provides the worst estimates, all other closures perform fairly well.

Example: We consider the stochastic logistic model with

$$
a_{1}=.30, a_{2}=.02, \quad b_{1}=.015, \quad b_{2}=.001,
$$

which corresponds to the population dynamics of the African Honey Bee [3]. Using (9), we have the following truncated moment dynamics

$$
\left[\begin{array}{c}
\dot{v}_{1} \\
\dot{v}_{2}
\end{array}\right]=\left[\begin{array}{rc}
0.28 & -0.016 \\
.32 & .546
\end{array}\right]\left[\begin{array}{l}
v_{1} \\
v_{2}
\end{array}\right]+\left[\begin{array}{r}
0 \\
-0.032
\end{array}\right] \varphi_{3}(v) \text {. }
$$

The time evolution of the moments corresponding to different moment closure techniques is obtained by substituting the appropriate moment closure function from Table II in place of $\varphi_{3}(v)$. In order to evaluate the performance of these moment closure functions for all time, we compute the exact evolution of the moments. This is only possible because the population limit $U=25$ is small and one can obtain the exact solution by numerically solving the Kolmogorov equation. Figures 1, 2 contains plots of the variance errors for the different moment closure functions with $x_{0}=5$ and $x_{0}=20$ respectively. The letters $s, g, p 1, p 2$ and $b$ are used to denote the errors corresponding to separable derivative-matching, normal, Nasell-poisson, New-poisson and binomial moment closure functions, respectively. For $x_{0}=20$ the binomial moment closure function provides the best estimate both initially and at steady-state, whereas for $x_{0}=5$ the New-poisson moment closure function does best initially, but the binomial moment closure function continues to provide the most accurate steady-state estimate. As one would expect from (27), the Nasell-poisson moment closure function performs the worst. Similar plots are obtained for the mean error $\mu_{1}(t)-v_{1}(t)$, which are not presented here due to lack of space.

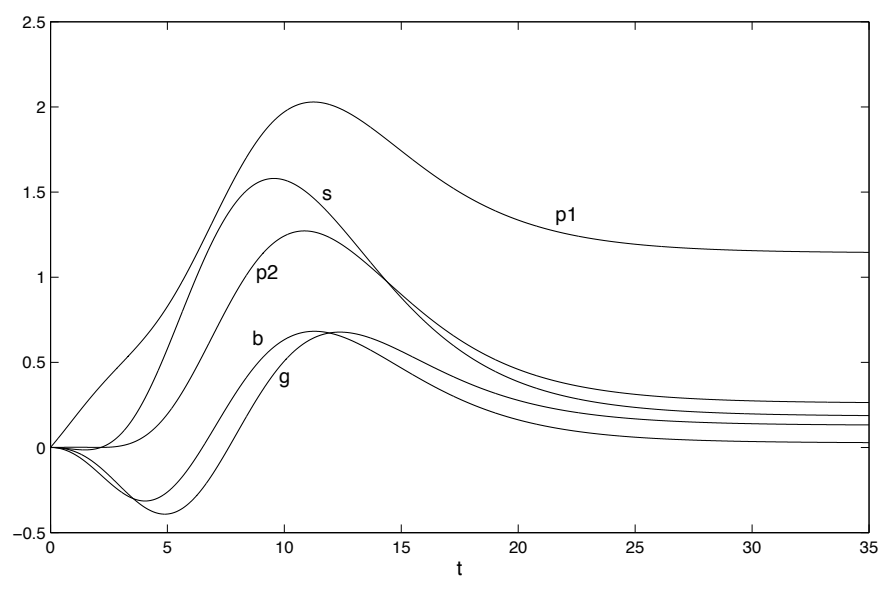

Fig. 1. Propagation of $\left[\left(\mu_{2}(t)-\mu_{1}(t)^{2}\right)-\left(v_{2}(t)-v_{1}(t)^{2}\right)\right]$ for different moment closures with $x_{0}=5$.

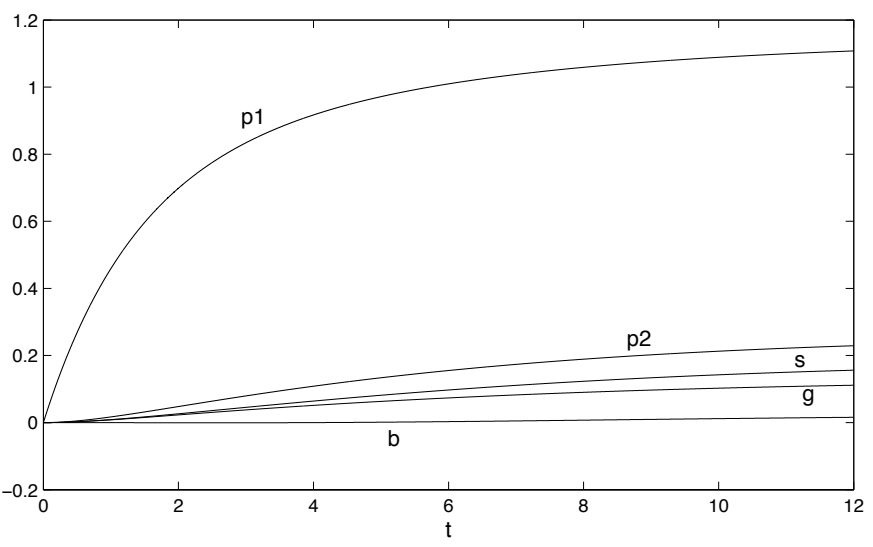

Fig. 2. Propagation of $\left[\left(\mu_{2}(t)-\mu_{1}(t)^{2}\right)-\left(v_{2}(t)-v_{1}(t)^{2}\right)\right]$ for different moment closures with $x_{0}=20$.

\section{B. Steady-state solutions}

Without going into any details we summarize the our result in this theorem. For details of the proof, the reader is referred to [13]. 
Theorem 3: Consider the stochastic logistic model (22). Then the truncated moment dynamics (14) with the separable derivative-matching moment closure function as given by Table I has a unique non-trivial attracting positive real steady-state for all order of truncations $n \geq 2$.

In terms of the parameters $a_{1}, b_{1}, a_{2}$ and $b_{2}$, the solutions for $n=2$ is

$$
v_{1}^{s}(\infty)=\frac{K}{1+\frac{\sigma^{2}}{K^{2}}}
$$

where

$$
K=\frac{a_{1}-a_{2}}{b_{1}+b_{2}}, \quad \sigma^{2}=\frac{a_{1} b_{2}+b_{1} a_{2}}{\left(b_{1}+b_{2}\right)^{2}} .
$$

Using Table II, we get the following non-trivial steady-state solutions for other moment closures and $n=2$ :

$$
\begin{aligned}
& v_{1}^{g}(\infty)=K\left[\frac{3}{4} \pm \frac{1}{4}\left(1-\frac{8 \sigma^{2}}{K^{2}}\right)^{\frac{1}{2}}\right] \\
& v_{1}^{p 1}(\infty)=K\left[\frac{3}{4} \pm \frac{1}{4}\left(1-\frac{8\left(\sigma^{2}-1\right)}{K^{2}}\right)^{\frac{1}{2}}\right] \\
& v_{1}^{p 2}(\infty)=\left[\frac{3 K-1}{4} \pm \frac{1}{4}\left((K+1)^{2}-\frac{8 \sigma^{2}}{K^{2}}\right)^{\frac{1}{2}}\right] \\
& v_{1}^{b}(\infty)=K-\frac{\sigma^{2}}{K-1} .
\end{aligned}
$$

From the above steady-states we conclude the following:

1) The binomial moment closure leads to a unique nontrivial attracting real steady-state, which can be negative for a range of parameters.

2) Normal, New-poison and Nasell-poison moment closures, yield two non-trivial steady-states, with the one with the - sign being a "spurious steady-state". Following the definition in [14], a steady state is "spurious", if $\lim _{M \rightarrow \infty} v_{1}(\infty) \neq K$, where $\sigma^{2}$ and $K$ are both $\mathscr{O}(M)$. For $n=2$, all these "spurious steadystates" happen to be un-stable, and hence, the truncated model will not converge to them. When the parameters are chosen, such that the term under the square root sign is negative, then both the non-tivial steady-states would be imaginary, which is biologically meaningless.

3) The separable derivative-matching moment closure leads to a unique non-trivial attracting positive real steady-state, and hence, with no spurious or imaginary steady-states has a clear advantage.

\section{CONCLusion And Future Work}

An approximate stochastic model for a continuous-time birth-death Markov processes was presented in this paper. This was done by representing the population of the specie as the continuous state of a SHS. With such a representation, the dynamics of the infinite vector containing all the statistical moments of the continuous state are governed by an infinite-dimensional linear system of ODEs. Finitedimensional nonlinear ODEs approximations were obtained by matching its time derivatives with those of the infinitedimensional ODEs at some time $t=t_{0}$. Explicit formulas to construct these finite-dimensional nonlinear ODEs were provided. Comparisons based on transient performance and steady-state solutions of the finite-dimensional ODEs were done, with alternative approximations available in literature, which lead to the following conclusion.

1) With the exception of the Nasell-poisson, all the moment closures achieve approximate derivative matching, and hence, give fairly good estimates. We proposed an alternative New-poisson moment closure, which unlike the Nasell-poisson moment closure proposed in literature, does match derivatives approximately, and thus performs better.

2) The separable derivative-matching moment closure, always yields a unique non-trival positive real steadystate $\forall n \in \mathbb{N}_{\geq 2}$, and hence, in some sense superior to the other moment closures, which can have spurious, imaginary and even stable negative steady-states.

A possible directions for future research is to extend the results of this paper to multi-specie birth-death Markov processes. Preliminary result regarding this appear in [9].

\section{REFERENCES}

[1] J. H. Matis and T. R. Kiffe, "On interacting bee/mite populations: a stochastic model with analysis using cumulant truncation," Enviromental and Ecological Statistics, vol. 9, pp. 237-258, 2002.

[2] — " "On apprroximating the moments of the equilibrium distribution of a stochastic logisitc model," Biometrics, vol. 52, pp. 155-166, 1996.

[3] J. H. Matis, T. R. Kiffe, and P. R. Parthasarathy, "On the cumulant of population size for the stochastic power law logisitc model," Theoretical Population Biology, vol. 53, pp. 16-29, 1998.

[4] N. T. J. Bailey, The Elements of Stochastic Processes. New York: Wiley, 1964.

[5] A. Singh and J. P. Hespanha, "Modeling chemical reactions with single reactant specie," in Proc. Workshop on Modeling and Control of Complex Systems, Cyprus, 2005.

[6] P. Whittle, "On the use of the normal approximation in the treatment of stochastic processes," J. Roy. Statist. Soc., Ser. B, vol. 19, pp. 268281, 1957.

[7] M. J. Keeling, "Multiplicative moments and measures of persistence in ecology," J. of Theoretical Biology, vol. 205, pp. 269-281, 2000.

[8] I. Nasell, "An extension of the moment closure method," Theoretical Population Biology, vol. 64, pp. 233-239, 2003.

[9] J. P. Hespanha and A. Singh, "Stochastic models for chemically reacting systems using polynomial stochastic hybrid systems," Int. J. of Robust and Nonlinear Control, vol. 15, pp. 669-689, 2005.

[10] J. P. Hespanha, "Stochastic hybrid systems: Applications to communication networks," in Hybrid Systems: Computation and Control, ser. Lect. Notes in Comput. Science, R. Alur and G. J. Pappas, Eds. Berlin: Springer-Verlag, Mar. 2004, no. 2993, pp. 387-401.

[11] — , "Polynomial stochastic hybrid systems," in Hybrid Systems : Computation and Control (HSCC) 2005, Zurich, Switzerland, 2005.

[12] E. C. Pielou, Mathematical Ecology. New York: Wiley, 1977.

[13] A. Singh and J. P. Hespanha, "Moment closure for the stochastic logistic model," Center for Control, Dynamical Systems and Computation. University of California at Santa Barbara, Tech. Rep. CCEC-06-0117, 2006, available at http://www.uweb.ucsb.edu/ abhi/papers/tech/moment.pdf.

[14] I. Nasell, "Moment closure and the stochastic logistic model," Theoretical Population Biology, vol. 63, pp. 159-168, 2003. 